\title{
Collector system of a gyrotron with magnetically shielded solenoid
}

\author{
M.V. Morozkin ${ }^{1}$, M.Yu. Glyavin ${ }^{1}$, V.N. Manuilov ${ }^{1,2}$, I.V. Zotova ${ }^{1}$ and M.D. Proyavin ${ }^{1}$ \\ ${ }^{1}$ Institute of Applied Physics of the Russian Academy of Sciences, N. Novgorod, Russia, morozkin@ipfran.ru \\ ${ }^{2}$ Lobachevsky State University of Nizhny Novgorod, N. Novgorod, Russia
}

The gyrotron-based complexes developed by IAP RAS are used now for many applications, such as sintering of nanostructured ceramics, metal compacts and metal-ceramic composites, CVD growth of diamond films and disks, and generation of multi-charged ion beams [1-3]. These systems typically operate in $24-28 \mathrm{GHz}$ frequency range with a power of $5-15 \mathrm{~kW}$. As a source of microwave radiation, the systems use $\mathrm{CW}$ gyrotrons operating in magnets with oil or water cooling (so called technological gyrotrons). To reduce power consumption of magnets, gyrotrons operate on second cyclotron harmonic.

Nowadays applications of gyrotron-based setups become more demanding in terms of output power. Also new applications appear that require high power, for example fabrication of metal oxides nanopowders [4] or production of oversized diamond disks for vacuum windows.

At present, a powerful gyrotron complex is being developed at the Institute of Applied Physics of the Russian Academy of Sciences, which includes a "warm" solenoid with magnetic shielding [5, 6] (see Fig. 1). Due to ferromagnetic screens, it is possible either to reduce the energy consumption of the solenoid in more than two times, or to increase the frequency of radiation while maintaining the energy of the magnetic system. The main parameters of the gyrotron are:

\begin{tabular}{|l|c|}
\hline Frequency $f$ & $28 \mathrm{GHz}$ \\
\hline Cyclotron harmonic $s$ & 1 \\
\hline Accelerating voltage $U_{0}$ & $23 \mathrm{kV}$ \\
\hline Operating current $I_{0}$ & $2.4 \mathrm{~A}$ \\
\hline Operating mode & $\mathrm{TE}_{1,3}$ \\
\hline Beam radius in the cavity $R_{0}$ & $6.6 \mathrm{~mm}$ \\
\hline
\end{tabular}

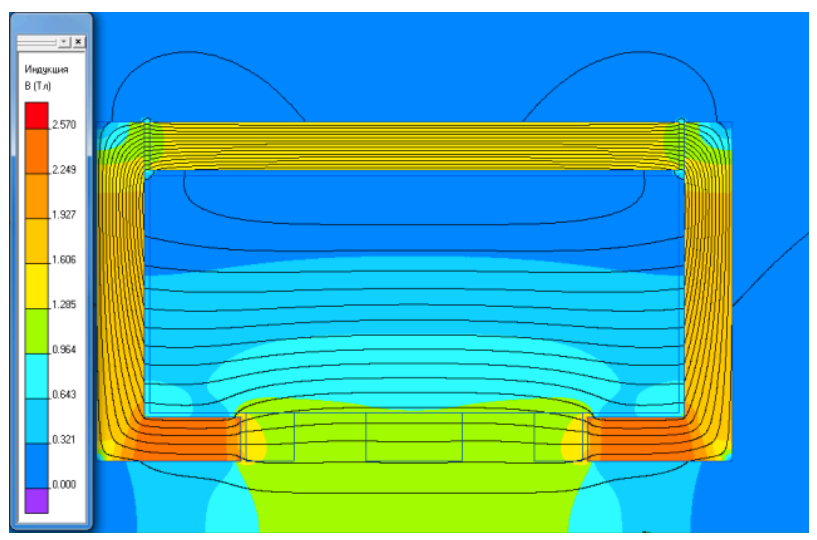

Fig. 1. The scheme of magnetic system with shielded solenoid. Magnetic field lines are shown

At the same time ferromagnetic screens substantially increases the inhomogeneity of the magnetic field outside the screens, in particular in the collector region. So here the coefficient of non-adiabaticity $\varepsilon=h / L_{B}$ (here $h$ is the step of electron trajectory, $L_{B}$ is the scale of the magnetic field) exceeds 2 (see Fig. 2).

Due to nonstandard distribution of the magnetic field in such systems and increased energy of an electron beam, collector of a gyrotron with magnetically shielded solenoid would differ significantly from collector of a standard technological gyrotron. The intensity of the magnetic field outside the shielding decays very rapidly; due to this, the motion of the electrons will be nonadiabatic. In this report, a collector design is presented and the simulation of the motion of electrons in the collector region is carried out.

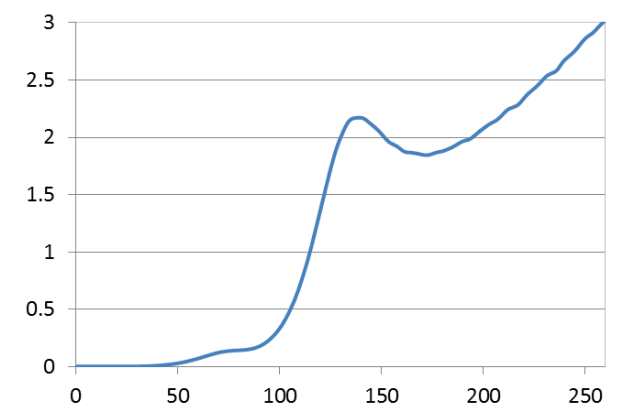

Fig. 2. Dependence of the coefficient of non-adiabaticity $\varepsilon$ on the longitudinal coordinate in the optimized magnetic system. $z=0$ corresponds to the cavity center

The results of the simulation (see Fig. 3) show that, due to the rapid decay of the magnetic field, electrons, falling into the region of the nonadiabatic field, acquire a transverse velocity and twist, starting to move downward along the radius. Then, when the magnetic field almost completely decays, the electron beam continues to fly apart inertially, while the direction of motion of the electrons is determined by the angle of the velocity vector at the exit from the region where the magnetic field is still significant.

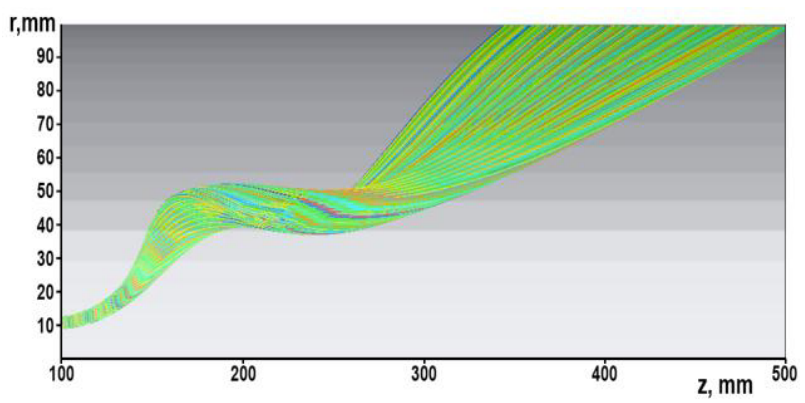

Fig. 3. Trajectories of the electron beam in the collector region (disregarding collector walls)

The inertial expansion of the electron beam provides a large ratio of the beam track length to the radius of the collector $R \mathrm{c}$ and a smooth energy density distribution within the track. However it is possible to use this effect 
effectively only at large collector radii, which may be relevant for a more powerful $(50-100 \mathrm{~kW})$ gyrotron.

For our gyrotron with a power of $20 \mathrm{~kW}$, the most optimal collector radius is $45 \mathrm{~mm}<R \mathrm{c}<50 \mathrm{~mm}$, at which part of the electrons will sit on the collector before the moment of twisting, and the part will fly further and descend already at the stage of inertial expansion. In this case, the total length of the electron beam trace will increase by $2-3$ times in comparison with the length even at a slightly larger radius $(R \mathrm{c} \sim 60)$, which will allow achieving low values of dissipated power density without using additional electron beam spreading systems.

Calculations show that the distribution of power density in the region of beam deposition is quite inhomogeneous and represents two peaks spaced in $\mathrm{z}$ (see Fig. 4). However, this calculation is made for the worst case in terms of load on the collector, when there is no generation. With the real spent electron beam, the power density distribution can become more homogeneous due to electrons with energies lower than the initial one.
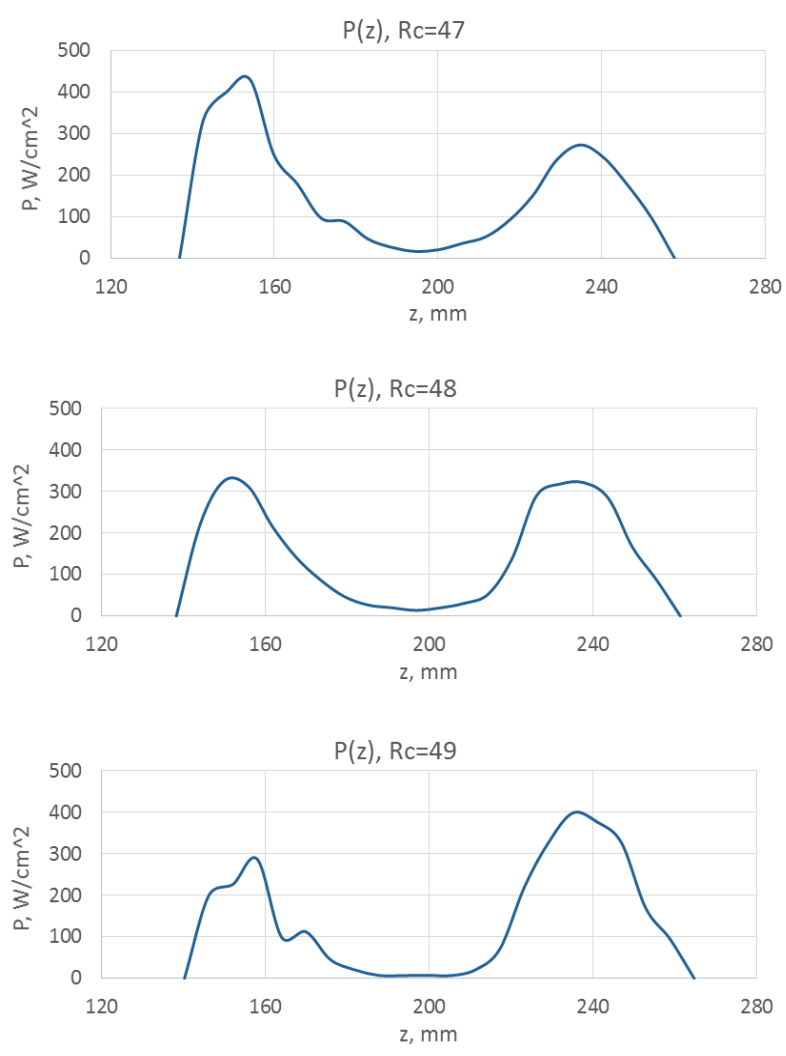

Fig. 4. Dependence of the power density on the collector on the $\mathrm{z}$ coordinate for different collector radii $(R \mathrm{c}=47,48$ and 49 from top to bottom)

The most optimal position of the collector wall is that at which part of the electrons sit down on the collector at the moment of twist, and the part flies further and settles down already at the stage of inertial expansion. In this case, the effective length of the track of the electron beam increases by 2-3 times, which makes it possible to achieve low values of the power dissipation at a relatively small radius of the collector without using additional systems for spreading the electron beam.

We determined the optimal radius of the collector to minimize the thermal load, and for a $20-\mathrm{kW}$ gyrotron, the maximum power density scattered on the collector was about $300 \mathrm{~W} / \mathrm{cm}^{2}$ (see Fig. 5), which leaves a reserve for increasing the gyrotron power with a shielded warm solenoid up to $40-50 \mathrm{~kW}$.

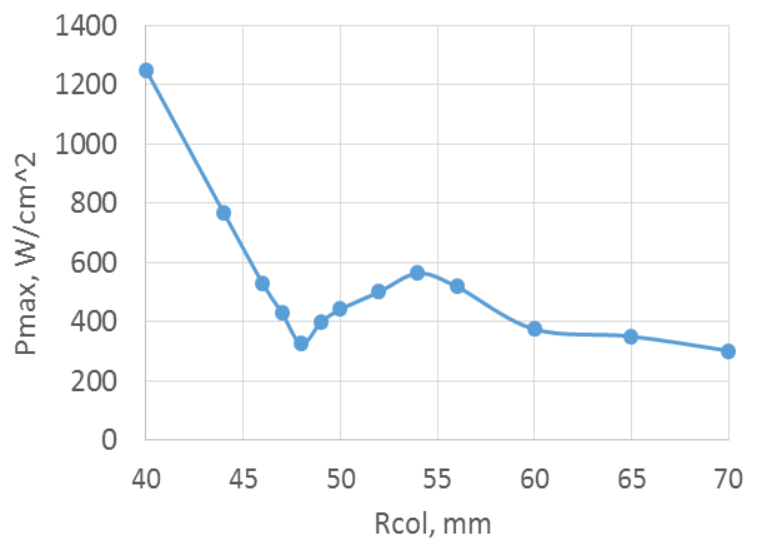

Fig. 5. Dependence of the maximum deposited power on the collector radius

\section{Conclusion}

The motion of electrons in the collector region of a gyrotron with a magnetically shielded solenoid was analyzed. A variant of the collector is proposed that uses the motion of electrons in the region of a nonadiabatic magnetic field and makes it possible to obtain a long trace of the deposited electron beam and a low power density on the collector without additional beam spreading systems.

\section{Acknowledgements}

This work was supported by the Russian Science Foundation, project No. 14-12-00887.

\section{References}

1. Bykov, Yu., Eremeev, A., Glyavin, M., Kholoptsev, V., Luchinin, A., Plotnikov, I., Denisov, G., Bogdashev, A., Kalynova, G., Semenov, V., Zharova, N. 24-84-GHz gyrotron systems for technological microwave applications // IEEE Trans. Plasma Sci. 2004, V. 32, No. 1, P. 67-72.

2. Vikharev, A. L., Gorbachev, A. M., Kozlov A. V., Koldanov, V. A., Litvak, A. G., Ovechkin, N.M., Radishev, D.B., Bykov, Yu.V., Caplan, M. Diamond films grown by millimeter wave plasma-assisted CVD reactor // Diam. Relat. Mater., 2006, V. 15 , No. 4-8, P. 502-507.

3. Bykov, Yu., Denisov, G., Eremeev, A., Gorbatushkov, V., Kurkin, V., Kalynova, G., Kholoptsev, V., Luchinin, A., Plotnikov, I. $28 \mathrm{GHz} 10 \mathrm{~kW}$ gyrotron system for electron cyclotron resonance ion source // Rev. Sci. Instr. 2004, V. 75, P. 1437.

4. Samokhin, A. V. , Alexeev, N. V., Vodopyanov, A. V., Mansfeld, D. A., Tsvetkov, Yu. $V$. Metal Oxide Nanopowder Production by Evaporation-Condensation Using a Focused Microwave Radiation at a Frequency of $24 \mathrm{GHz} / / \mathrm{J}$. Nanotechnol. Eng. Med. 2015, V. 6, No. 1, P. 011008.

5. Kuftin, A.N., Belov, S.P. Magnetic shielded electron optical system of a gyrotron in space charge limited current regime // Applied Physics 2000, No. 3, P. 76-81.

6. Glyavin, M. Yu., Manuilov, V. N., Proyavin, M. D. Electron optics system of continuous wave gyrotron with an operating frequency $24 \mathrm{GHz}$ based on shielded solenoid" // J. Commun. Technol. Electron. 2017, submitted. 